Innovaciones de Negocios 12(23): 113 - 131

(c) 2015 UANL, Impreso en México (ISSN 2007-1191)

Fecha de recepción: 29 de mayo de 2015. Fecha de aceptación: 12 de junio de 2015

\title{
Rol y mejores prácticas del equipo líder y su impacto en la innovación de empresas de la Zona Metropolitana de Monterrey
}

\section{(Role and best practices of the leadership team and its impact on business innovation in the metropolitan area of Monterrey)}

\author{
Laura Elizabeth Avila Hernández \& Gustavo Juan Alarcón Martínez \\ Universidad Autónoma de Nuevo León, Facultad de Contaduría Pública y Administración, \\ San Nicolás de los Garza, N.L., México. \\ Email: laura.avila@ccmty.com y laura.avila@gmail.com
}

Key words: high management teams, innovative company, key success factors, leadership JEL: 030 \& M10

\begin{abstract}
This research article elaborates on the leadership or high management teams that have taken into account the innovation challenges within their organizations and the best practices carried out by them. In that token some business innovation definitions are depicted, as well as findings by business researchers on best innovation practices conducted namely by leadership or high management teams. Lastly, the regression analysis results and conclusions are show.
\end{abstract}

Palabras clave: alta dirección, empresa innovadora, factores clave de éxito, liderazgo JEL: 030 \& M10

Resumen. Este artículo es una investigación sobre las prácticas que realiza la alta administración o el equipo líder de empresas que han asumido el reto de la innovación en sus organizaciones. Para tal efecto se presentan algunas definiciones de empresa innovadora, posteriormente, se mencionan los roles o prácticas que han encontrado destacados investigadores en empresas que han desarrollado innovación; particularmente se analizan las prácticas que realiza el equipo líder o la alta administración. Finalmente se presenta los resultados de un análisis de regresión simple con sus respectivas conclusiones. 


\section{Introducción}

Las empresas actualmente enfrentan diversos desafíos como la sustentabilidad, la competitividad, los cambios tecnológicos, la situación económica y financiera nacional e internacional; existen también otros elementos al interior de las organizaciones como el capital humano, la infraestructura, la sistematización de procesos, la atención a los clientes, la cadena de suministros, que hacen que sea compleja y retadora la administración exitosa de una empresa. Sin lugar a duda en quien recae la responsabilidad de orientar el rumbo de la organización para lograr su sustentabilidad y competitividad en el corto, mediano y largo plazo es en el equipo líder o conocido también como la alta administración de la empresa.

Ante estos retos que enfrentan los líderes de las organizaciones es relevante considerar y aprender las mejores prácticas que están realizando las altas administraciones de las empresas que están compitiendo en forma exitosa en la contienda global.

Cabe mencionar que el World Economic Forum considera a la innovación como uno de los doce pilares que conforman la competitividad de las regiones; también se reconoce que son las empresas como los agentes del desarrollo económico y competitividad de los países (OCDE, 2006). Así se vuelve prioritario estudiar las prácticas características de los equipos líderes de las empresas innovadoras que impulsan a las organizaciones a mejores niveles de crecimiento y desarrollo sustentable.

Este artículo está basado en una investigación previa realizada sobre los factores críticos de éxito de las empresas innovadoras de la Zona Metropolitana de Monterrey (ZMM). En el presente artículo se busca presentar con mayor detalle las prácticas realizadas por los equipos líderes y su impacto en los resultados de innovación de empresas de la ZMM.

Al respecto se presentan algunas definiciones de empresa innovadora así como el enfoque de la innovación en las mismas. Posteriormente se mencionan las prácticas encontradas por diferentes autores derivadas de las investigaciones que realizaron en empresas que han desarrollado innovación. Finalmente se exponen los resultados obtenidos para el caso de empresas de la ZMM y se presentan conclusiones de la investigación realizada. 


\section{Marco de teórico}

En la actualidad la sociedad y las empresas que la conforman están inmersas en un entorno marcado por la globalización, la competencia, los cambios tecnológicos, la información a través de las redes sociales, la responsabilidad social y ecológica, así como una profunda crisis económica internacional; por lo anterior, las empresas necesitan desarrollar técnicas de dirección y administrar procesos que les permitan alcanzar la competitividad (Instituto Mexicano de Ejecutivos de Finanzas, A.C., 2010).

Como se ha señalado con antelación, el World Economic Forum (2012) ha distinguido que la innovación es uno de los 12 pilares que constituyen a la competitividad. En el 2008 la Cámara de la Industria de la Transformación de Nuevo León (CAINTRA NL) a través del Centro de Competitividad de Monterrey (CCM) realizó un estudio en 288 Pequeñas y Medianas Empresas de la Zona Metropolitana de Monterrey. En dicho estudio se analizaron los elementos que constituyen la competitividad de las empresas, encontrándose que una de las características de las empresas de éxito es la innovación y la mejora continua.

La experiencia internacional demuestra que las empresas son los agentes principales de la innovación. Según los estudios realizados por el CONACYT en México, se observa que existen esfuerzos del sector privado por incrementar sus capacidades para la generación de la innovación buscando mejorar su competitividad; sin embargo, estos esfuerzos se han concentrado en algunas empresas y ramas industriales y no se ha generalizado por lo que existe una oportunidad para desarrollar sistemas de gestión con base en experiencias y prácticas exitosas que favorezcan el desarrollo de las empresas (Comité Intersectorial para la Innovación, 2011).

\section{Enfoque sobre innovación empresarial}

Joseph Schumpeter (1966) es el primero que describe la innovación como motor de desarrollo económico y también porque la considera como un proceso dinámico continuo, donde las nuevas tecnologías desplazan a las antiguas. Otra aportación de Schumpeter es la clasificación que hizo sobre los tipos de innovación: introducción de nuevos productos; introducción de nuevos métodos de producción; apertura de nuevos mercados; desarrollo de 
nuevas fuentes de abastecimiento de materias primas u otros recursos; creación de nuevas estructuras de mercado. En el Manual de Oslo (2006) la OCDE señala que las innovaciones pueden ser: "radicales" cuando implican un cambio relevante; y las "incrementales" cuando son innovaciones que mejoran en forma continua el proceso o producto, esto es son mejoras dentro del mismo paradigma (OCDE, 2006) .

Según Drucker (1985) el proceso de innovación debe estar orientado al cliente e identifica tres enfoques: con base en las necesidades percibidas del cliente, la innovación enfocada en las necesidades no percibidas del cliente y la innovación orientada por las necesidades futuras de los clientes (Garza Villegas, 2009).

Thomas Kuczmarski (1997) considera la innovación como una responsabilidad y forma de pensar de los líderes, la cual implica directrices y acciones de la alta administración de la empresa.

Allan Afuah (1999) presenta a la innovación como el uso de "conocimiento tecnológico y de mercado" el cual es utilizado por la empresa para desarrollar y brindar un nuevo producto o servicio a sus clientes. También Afuah considera que la innovación puede tener dos vertientes: ser técnica o administrativa; puede ser algo nuevo pero no necesariamente un producto material, puede ser también un cambio fundamental en alguna práctica administrativa o proceso.

Para Clayton M. Christensen (1999) la innovación está en estrecha relación con la tecnología describiéndola como los procesos por los cuales una organización transforma el trabajo, el capital, los materiales y la información que recibe, en productos y servicios de mayor valor para el cliente o mercado.

Ernest Gundling (2000) hace referencia a los tres distintos tipos de innovación que realiza $3 \mathrm{M}$ a saber: innovación de tipo radical esto es la que da a luz a un nuevo negocio o industria; el segundo tipo se relaciona con innovaciones que crean una nueva posición competitiva o nicho de mercado; el tercer tipo se asocia con la extensión de línea de producto, con aquellos productos y servicios que tienen un avance incremental.

En el 2003 Henry Chesbrough acuña el término "innovación abierta" (open innovation); el cual se fundamente en la idea de que las empresas ya no son capaces de abordar por sus propios medios todo el proceso de la 
innovación; Chesbrough desarrolla el concepto de que las empresas deben establecer relaciones con fuentes externas (propiedad intelectual, ideas, productos, personas, instituciones) las cuales deben integrarse en su propia cadena de innovación.

Luigi Valdés (2004) define el concepto de innovación como "Innovación es el arte de crear algo nuevo, novedoso y original, colocarlo en un mercado y que el cliente esté dispuesto a pagar por ello". De acuerdo a Valdés la innovación es un proceso sistémico orientado al cliente y para que este sea completo tiene que lograr un impacto en el mercado y esto dependerá en gran medida de los niveles de la innovación, los cuales pueden ser graduales 0 evolutivos, de rompimiento o de arquitectura de nuevos modelos de negocio.

Daniel Goleman (2009) autor de la inteligencia emocional, describe la innovación empresarial como la creatividad en el lugar de trabajo, como una capacidad para la creación de nuevos productos y de mejores servicios a sus clientes; es una capacidad para introducir innovaciones en la administración mejorando los métodos de distribución o aplicando ideas nuevas para financiar el negocio.

Fernando Trías de Bes y Philip Kotler (2011) hacen la siguiente afirmación "Por innovación debe entenderse también el desarrollo de una cultura innovadora en la empresa, la cual hará posible que produzca y ponga en el mercado una corriente continua de innovaciones menores o marginales. Es entonces cuando, aunque parezca paradójico, la innovación radical acabará surgiendo." También afirman "es muy difícil, por no decir imposible, que una organización que lanza pocas innovaciones marginales sea exitosa lanzando una innovación radical. En un caso así, la cultura de innovación no estará suficientemente desarrollada y la empresa no se encontrará probablemente preparada para ejecutarla. Una empresa que no está acostumbrada a innovar de forma regular no suele hacerlo bien cuando ha de innovar de forma excepcional".

Considerando las descripciones de los autores anteriormente citados se puede observar que para describir a la innovación empresarial existen elementos en común, algunos de ellos son: nuevos productos, nuevos procesos, nuevas metodologías, desarrollo tecnológico y la creación de valor para el mercado o los clientes. 


\section{Características de la innovación.}

Al realizar la revisión sobre innovación en diferentes ámbitos se observa que puede ser de tres tipos: competencias de las personas innovadoras; características de las empresas innovadoras; así como políticas y estrategias de países o regiones innovadoras. La presente investigación se enfoca a la descripción del rol o prácticas del equipo líder en las empresas innovadoras por lo cual a continuación se presenta una breve descripción de ambos términos.

Empresa Innovadora.

Para definir dicho concepto, se presenta en primera instancia dos definiciones básicas: empresa e innovación.

De acuerdo al CONACYT se considera como empresa a "persona moral que realiza actividades económicas dirigidas a la producción, distribución o al intercambio de bienes o servicios para el mercado con fines de lucro; así como las instituciones sin fines de lucro, que están esencialmente al servicio de la empresa" (CONACYT, 2008).

En cuanto a innovación, etimológicamente el término de innovar proviene del latín innovare, que significa cambiar o alterar las cosas introduciendo novedades (RAE, 2013).

El CONACYT en "Bases de Organización y Funcionamiento del Registro Nacional de Instituciones y Empresas Científicas y Tecnológicas (RENIECYT)", describe la innovación tecnológica de la siguiente manera: "Conjunto de actividades ordenadas que conducen a la obtención e implementación de nuevos productos y procesos, así como a lograr cambios significativamente mejorados en los mismos" (CONACYT, 2008).

El Manual de Oslo define la empresa innovadora como "aquella que ha introducido una innovación durante el periodo de tiempo considerado. Las innovaciones no necesitan haber sido un éxito comercial, muchas innovaciones fracasan. Las empresas innovadoras se dividen en las que principalmente han desarrollado innovaciones por sí mismas 0 en cooperación con otras empresas u organizaciones de investigación públicas, o las que han innovado principalmente adoptando las innovaciones 
desarrolladas por otras empresas. Las empresas innovadoras se pueden distinguir también por el tipo de innovación que han introducido, si han desarrollado un nuevo proceso o producto, o si han introducido un nuevo método de comercialización o un cambio en la organización" (OCDE, 2006).

Dados los conceptos anteriormente expuestos, se considera como empresa innovadora aquella que ha introducido una innovación durante el periodo de tiempo considerado.

Equipo líder o alta administración.

Existe una vasta literatura sobre el liderazgo y sus diferentes enfoques, se puede encontrar definiciones y estudios sobre liderazgo político, liderazgo económico, liderazgo empresarial, liderazgo religioso, entre otros. Dada la orientación del presente artículo, se consideran las aportaciones de diversos autores en la descripción de las características del liderazgo que tienen las empresas para implementar estrategias que fomenten la innovación en sus organizaciones.

Una de las características de los líderes es su característica de estratega para desarrollar planes, estrategias y directrices (Westcott, 2006). Kuczmarski (1997) afirma que la innovación inicia por la alta gerencia puesto que es ella la que establece las estrategias y las acciones que orientan la innovación en toda la empresa. También Afuah (1999) identifica la estrategia de la empresa como un elemento crítico que trazará el rumbo de la innovación. Fernando Trias de Bes y Philip Kotler (2011) afirman lo siguiente: "La planeación es el primero de los elementos de un Sistema Integral de Innovación, planificar permite alinearse con la estrategia corporativa, asignar recursos de modo eficiente y alimentar ordenadamente la planeación operativa para los procesos de innovación de la compañía. La planeación de la innovación es responsabilidad conjunta del $\mathrm{CIO}$ y del CEO, o de la alta gerencia de la empresa dado que se trata de un área estratégica y crítica para la supervivencia de la organización".

Un equipo de más de 30 investigadores del Minnesota Innovation Research Program (MIRP), en su publicación identificaron cuatro tipos de liderazgo: el Patrocinador, el Mentor, el Crítico y el Líder institucional, (Van de Ven, et al. 2000). 
Otra característica del liderazgo es el rol como administrador que organiza y optimiza el uso de los recursos tanto de capital humano como de capital de trabajo (Westcott, 2006). Kuczmarski (1997) describe la relevancia de una organización holística para la innovación, lo cual implica contar con equipos de trabajo interfuncionales responsables de innovar, establecer un sistema de reconocimiento, contar normas y valores que guíen el comportamiento del personal, así como establecer un proceso de desarrollo por etapas, lo que permite que los equipos de innovación cuenten con una estructura y guía para transformar una nueva idea en un producto real.

Clayton Christensen (1999) en su investigación sobre innovaciones disruptivas habla sobre los errores que puede cometer la alta administración y los principios que deben considerarse para no fracasar; entre ellos se destaca la relevancia que tiene para la empresa los mercados actuales y la creación de nuevos negocios con las tecnologías disruptivas desarrolladas.

Otro elemento común en el liderazgo estratégico para la innovación es el seguimiento que se realiza a través de indicadores. Kuczmarski (1997) y Trias de Bes (2011) mencionan la importancia de contar con un sistema de indicadores para medir el progreso, la rentabilidad de la innovación y el impacto en la empresa.

Dado lo anteriormente expuesto se considera que el equipo líder o la alta administración realiza prácticas tales como: el establecimiento de directrices, estrategias, procesos, y mecanismos para orientar a toda la organización hacia el desarrollo sustentable basado en innovación.

\section{Prácticas y roles del equipo líder (o alta administración) para la gestión de la innovación en las empresas}

Russel T. Westcott (2006) distingue los roles y responsabilidades de los líderes, distingue diversos estilos de liderazgo como el liderazgo transaccional, el transformacional, el situacional, los líderes de opinión por mencionar algunos. En cuanto a los roles que realiza el equipo líder menciona los siguientes:

- Estratega que desarrolla directrices que orientan a la empresa en sus planes estratégicos así como en el despliegue en planes operativos, metas y objetivos. 
- Arquitecto que construye una estructura organizacional que soporta las estrategias, metas y objetivos.

- Administrador que organiza y optimiza el uso de los recursos tanto de capital humano como de capital de trabajo.

- Emprendedor porque genera nuevos negocios para la sustentabilidad de la organización.

- Innovador porque continuamente busca caminos para introducir nuevos productos, mejorar los procesos o llegar a nuevos mercados.

- Coach-entrenador, él mismo es guía y ejemplo, conoce y enseña sobre las actividades a realizar.

En cuanto a las responsabilidades de los líderes administrativos Westcott (2006) señala que son:

- La planeación estratégica, con todas sus implicaciones como el mapeo del despliegue en objetivos, metas, acciones, proyectos, inversiones.

- El diseño de la organización asegurando que es adecuada para la consecución de su misión, estrategias y metas, estableciendo su estructura organizacional con sistemas y procedimientos.

- La administración de capital humano, con funciones como la selección, el desarrollo de competencias y su retención creando un ambiente motivado en el trabajo para el logro de las estrategias.

- La dirección proveyendo el soporte y las acciones requeridas para el logro de los objetivos y metas.

- El control, monitoreando el desempeño contra los resultados planeados, tomando las acciones correspondientes para el logro de los mismos, identificando las áreas de mejora (Westcott, 2006).

Otro autor que describe el liderazgo empresarial es Thomas Kuczmarski (1997) quien afirma que la innovación comienza por la alta gerencia, porque es ella quien establece las estrategias y las acciones que orientan el desarrollo de la innovación en toda la empresa, también menciona que es responsabilidad de la alta gerencia establecer los indicadores para medir los esfuerzos, inversiones y resultados de la innovación en la organización. En su texto "Innovación: Estrategias de liderazgo para mercados de alta competencia" describe con precisión como el liderazgo de la alta gerencia debe ser comprometido a través de la creación de tres plataformas sobre las cuales se desarrollan los bloques de la innovación: 
- Planear para la innovación, que a su vez implica crear una visión, desarrollar una estrategia y diseñar un portafolio de tecnología e innovación;

- Definir el proceso de desarrollo, lo cual significa diseñar un proceso de desarrollo de la innovación por etapas: exploración de las necesidades del consumidor, generación de ideas, desarrollo de ingeniería conceptual, análisis del negocio, desarrollo de prototipo y pruebas en planta, prueba piloto en mercado entre otras;

- Formar una organización holística para la innovación, lo cual implica contar con equipos de trabajo interfuncionales responsables de innovar, establecer un sistema de reconocimiento, contar normas y valores que guíen el comportamiento del personal, finalmente contar un sistema de medición para medir el progreso y rentabilidad.

Otro investigador que identifica el liderazgo estratégico es Alan Afuah (1999), describiendo su rol en la toma de decisiones que realiza la alta dirección para invertir en el desarrollo de una innovación o para adoptarla. Esta toma de decisiones depende de dos elementos, un elemento son las creencias, valores y directrices de la empresa y el otro elemento es la capacidad de la alta gerencia de reconocer el potencial de la innovación en la industria en la cual se mueve la empresa. Afuah identifica la estrategia como un elemento clave y la define como una capacidad para recopilar y procesar información que la empresa puede seguir diversos tipos de estrategias entre ellas destaca dos, la estrategia de tipo ofensiva y la estrategia de tipo defensiva.

- La estrategia de tipo ofensiva es cuando la empresa quiere ser la primera en introducir nuevos productos, por lo cual sus acciones están dirigidas hacia la generación de nuevas ideas para convertirlas en productos antes de que lo hagan los competidores, para lograrlo invierten grandes cantidades en investigación y desarrollo.

- La estrategia de tipo defensiva, es la estrategia que siguen las empresas que esperan que una precursora introduzca primero el producto, y después introduce los propios corrigiendo errores que pudo cometer la empresa precursora, también estas empresas invierten en investigación y desarrollo ya que sus productos deben ser una imitación diferenciada; 
otros tipos de estrategias que pueden seguir las empresas son dependiente, tradicional y oportunista. (Afuah, 1999)

Clayton Christensen (1999) en su investigación sobre innovaciones disruptivas habla sobre los errores que pueden tener la alta administración, y al realizar su estudio identifica cuatro principios que debe considerar para no fracasar, estos son:

- Las empresas dependen de sus clientes e inversiones para obtener recursos y la alta administración debe considerar realizar nuevos negocios "spin off" con las nuevas tecnologías emergentes que se desarrollan en las empresas.

- Los mercados pequeños no resuelven las necesidades de crecimiento de las empresas grandes, la experiencia es que la alta administración típicamente asigna a otra organización la responsabilidad de comercializar los nuevos productos según el tamaño de su mercado.

- $\quad$ No se pueden analizar aquellos mercados que aún no existen, conocer el mercado es responsabilidad de una buena administración, sin embargo menciona Christensen, este es el dilema de los innovadores porque no se pueden estimar proyecciones financieras cuando no se conocen los ingresos y sus costos, estas estimaciones serán realizadas un tanto en el ejercicio de prueba y error.

- La provisión de tecnologías puede no ser igual que la que demanda el mercado. (Christensen C. M., 1999)

Un equipo de más de 30 investigadores del Minnesota Innovation Research Program (MIRP) publican su investigación sobre innovación, en dicha publicación identifican cuatro tipos de liderazgo:

- Patrocinador, el cual ocupa una posición suficientemente alta para ejercer el poder y disponer de los recursos necesarios para poner en práctica ideas innovadoras, él es quien gestiona la innovación entre la corporación y realiza roles como procuración, defensa, y representación en los niveles más altos de la organización.

- Mentor, típicamente este tipo de liderazgo es desempeñado por un innovador experimentado y exitoso, por lo cual incluye actividades de dirección, asesoría y consejo con los emprendedores de innovaciones sobre una base directa y cotidiana.

- Crítico, este tipo de liderazgo es el conocido como "abogado del diablo" por las actividades que desempeña ante la corporación y los 
emprendedores en cuanto a su actuar de cuestionar las inversiones, las metas o los avances de los proyectos de innovación.

- Líder institucional, se ocupa de conservar un equilibrio de poder entre las influencias favorables a la innovación y el influjo inquisitivo del crítico, además el líder institucional toma el rol de facilitador ante cambios de estructura o estilos administrativos, busca típicamente que los conflictos se resuelvan con base en los méritos del caso. (Van de Ven, et al. 2000)

En los estudios realizados por Fernando Trias de Bes y Philip Kotler (2011) afirman lo siguiente: "La planeación es el primero de los elementos de un Sistema Integral de Innovación, planificar permite alinearse con la estrategia corporativa, asignar recursos de modo eficiente y alimentar ordenadamente la planeación operativa para los procesos de innovación de la compañía. La planeación de la innovación es responsabilidad conjunta del $\mathrm{CIO}$ y del CEO, o de la alta gerencia de la empresa dado que se trata de un área estratégica y crítica para la supervivencia de la organización".

Con base en los autores y las definiciones anteriormente expuestas, se considera para la presente investigación que el liderazgo estratégico orientado a la innovación comprende: el establecimiento de directrices, estrategias, procesos y prácticas que la dirección debe establecer para orientar a toda la organización hacia el desarrollo sustentable basado en innovación; por lo cual es considerado uno de los factores críticos de éxito de las empresas innovadoras y una variable independiente de la presente investigación.

\section{Planteamiento del problema}

Uno de los principales problemas que enfrenta México es su desarrollo económico, el cual radica en la capacidad de generación de riqueza de forma particular por parte de sus empresas. Es por ello que se vuelve relevante aprender de las experiencias de otras organizaciones que han logrado ventajas competitivas y que les ha permitido un crecimiento económico equiparable o mayor que el de sus pares (Comité Intersectorial para la Innovación, 2011). 
Al revisar el estado del arte sobre los elementos característicos del equipo líder o la alta administración de las empresas innovadoras, se encontró que existen estudios sobre las prácticas y roles de sus líderes en empresas de Estados Unidos y Europa, sin embargo en México se carece de estudios del impacto que tienen estas prácticas en su conjunto sobre los resultados en las empresas innovadoras.

\section{Objetivo de la investigación}

Analizar y determinar las prácticas y roles del equipo líder que impactan positivamente en los resultados de la innovación de procesos de la empresa innovadora de la Zona Metropolitana de Monterrey.

\section{Hipótesis de la investigación}

El liderazgo estratégico de la alta administración está relacionado de manera positiva con los resultados en innovación de las empresas innovadoras.

La ecuación de regresión se expresa matemáticamente mediante la siguiente ecuación:

$$
Y=\beta_{0}+\beta_{1} X+\varepsilon
$$

Donde:

$Y=$ Empresa innovadora (resultados en innovación)

$X=$ Liderazgo estratégico

Figura 1: Modelo de liderazgo estratégico de la alta administración

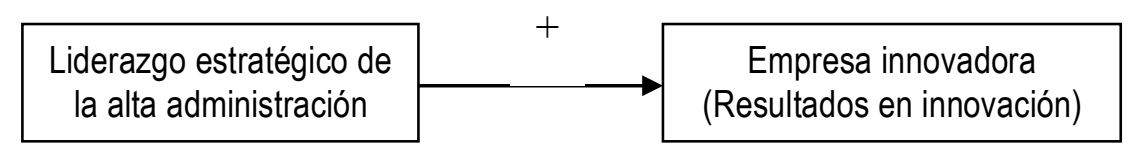

Fuente: Elaboración propia 
Operacionalización de la variable dependiente

El Manual de Oslo en el punto 47, define la empresa innovadora como aquella que ha introducido una innovación durante el periodo de tiempo considerado. También en el Manual de Oslo en el punto 48 se menciona que los impactos de las innovaciones en los resultados de la empresa pueden percibirse en las ventas 0 en la cuota de mercado $u$ originar cambios en la productividad y la eficiencia (OCDE, 2006, pág. 27). En términos de ítem significa:

- Resultado de la innovación en procesos considerado como el promedio del porcentaje de incremento en la productividad (o eficiencia), en los últimos tres años.

\section{Operacionalización de la variable independiente}

De acuerdo a lo descrito en el marco teórico, el liderazgo estratégico orientado a la innovación comprende el establecimiento de directrices, estrategias, procesos y prácticas que la dirección debe establecer para orientar a toda la organización hacia el desarrollo sustentable basado en innovación. Implica en términos de ítems:

- Plan estratégico que contiene visión, directrices, objetivos, metas y portafolio de nuevos productos o tecnologías;

- Diseño de estructura organizacional para el desarrollo de la innovación a través de manual de organización, con valores, normas, procedimientos;

- Selección e integración de equipos de trabajo con personal competente en diferentes funciones;

- Diseño e implementación de proceso de innovación con procesos, métodos documentados que conlleven a la innovación de productos 0 procesos;

- Diseño o implementación de proceso de innovación con procesos de vinculación con el mercado y con otras organizaciones (empresas, centros de investigación, universidades) para el desarrollo de nuevos productos o tecnologías; 
- Sistema de información con procedimientos y métodos para mantener la comunicación con el mercado (para identificación de necesidades, expectativas de clientes y usuarios);

- Inversión o asignación de recursos económicos o financieros para la innovación (presupuesto, fondos gubernamentales, créditos);

- Sistema de seguimiento al avance de los proyectos de innovación.

Para calcular el valor de la variable independiente por empresa considerando que está compuesta por 8 ítems, se siguió el método de promedios ponderados, donde el factor considerado para la ponderación es el "corrected item-total correlation" el cual se obtuvo a través del software SPSS, esta herramienta fue la utilizada para el análisis de los datos.

\section{Diseño de la investigación}

La presente investigación está basada en el método científico en forma cuantitativa y correlacional de acuerdo a las definiciones en Hernández Sampieri (2010). El instrumento de medición utilizado para la obtención de la información fue mediante una encuesta aplicada a empresas de la ZMM que han realizado innovación en sus productos, servicios o procesos en los últimos 5 años.

Para la medición de la variable dependiente el indicador considerado fue: promedio del porcentaje de incremento en la productividad (o eficiencia), en los últimos tres años. Para la variable independiente se utilizó la escala de Likert, donde el encuestado califica cada ítem según su apreciación en puntuaciones del 1 a 5, siendo: $1=$ Nada de acuerdo o no se cuenta; 2= Poco de acuerdo; 3= De acuerdo; 4= Muy de acuerdo; 5= Totalmente de acuerdo 0 casi completa.

Para establecer el tamaño de la muestra se consideraron las empresas que han recibido fondos del CONACYT para realizar proyectos de innovación y desarrollo tecnológico en sus organizaciones en los últimos 5 años, el número de organizaciones es de 96 empresas de la ZMM.

Considerando los principios estadísticos y realizando los cálculos correspondientes la "n optima" es de 48 empresas, en este cálculo se considera un error de estimación (nivel de precisión) equivalente al 0.1 esto 
es un $10 \%$, lo cual es considerado como apropiado en las ciencias administrativas.

En la aplicación del instrumento se logró recabar información de 43 encuestas que están realizando innovación con enfoque en sus procesos de operación; para analizar la confiabilidad del estudio se aplicó el alpha de Cronbach a las respuestas obtenidas y el resultado fue de 0.891 lo cual muestra que sus resultados son confiables.

\section{Presentación de resultados}

Con la intensión de mostrar estadísticamente la relación directa que existe entre las dos variables anteriormente descritas, se aplicó el análisis de regresión simple introduciendo los resultados de las encuestas en el SPSSTM obteniéndose la siguiente información. El resultado del modelo fue significativo con un valor de .000 , con una $F$ de 75.699 y con 42 grados de libertad (véase Tabla 1).

Tabla 1. ANOVA

\begin{tabular}{lcrccc}
\hline \multicolumn{1}{c}{ Modelo } & Suma de cuadrados & gl & Media cuadrática & F & Sig. \\
\hline Regresión & 10387.648 & 1 & 10387.648 & 75.699 & $.000^{\mathrm{b}}$ \\
Residual & 5626.154 & 41 & 137.223 & & \\
Total & 16013.803 & 42 & & & \\
\hline
\end{tabular}

a. Variable dependiente: YProcesos

b. Variables predictoras: (Constante), LidEst

La $R$ alcanzó un valor de 0.805 y el $R^{2}$ de 0.649 con un error de estimación de 11.714, considerándose como aceptable en las ciencias sociales, porque significa que la variable dependiente y su varianza es explicada en un $65 \%$ como se muestra en la Tabla 2.

Tabla 2. Resumen del modelo

\begin{tabular}{cccc}
\hline $\mathbf{R}$ & $\mathbf{R}$ cuadrado & $\mathbf{R}$ cuadrado corregida & Error típ. de la estimación \\
\hline $.805^{\mathrm{a}}$ & .649 & .640 & 11.714 \\
\hline
\end{tabular}

a. Variables predictoras: (Constante), Liderazgo Estratégico

Fuente: Elaboración propia con base en IBM SPSSTM 
Un indicador a observar en la regresión lineal es el Durbin Watson, el resultado obtenido es de 1.677 con el cual se puede asumir independencia entre los residuos porque su valor obtenido está entre 1.5 y 2.5 (Pardo Merino \& Ruiz Díaz, 2009)

Otro de los indicadores es el de FIV (Factor de Inflación de Varianza) el cual se recomienda sea menor a 4.0 (De la Garza García, Morales Serrano y González Cavazos 2003) y el resultado obtenido es de 1.00.

Finalmente la ecuación de regresión sería conforme a los datos obtenidos en la Tabla 3.

Tabla 3. Coeficientes

\begin{tabular}{lccccc}
\hline \multicolumn{5}{c}{ Coeficientes no estandarizados } & \multicolumn{2}{c}{ Coeficientes tipificados } \\
& $\mathrm{B}$ & Error típ. & Beta & $\mathrm{t}$ & Sig. \\
\hline (Constante) & 8.791 & 8.159 & & 1.077 & .288 \\
LidEst & 17.753 & 2.040 & .805 & 8.701 & .000 \\
\hline
\end{tabular}

Fuente: Elaboración propia con base en IBM SPSS ${ }^{\top M}$

$$
\text { YProcesos }=8.791+17.753 \text { LidEst }
$$

Cabe mencionar que el motivo por el cual no se considera utilizar el coeficiente tipificado es porque en el presente estudio solamente se tiene una variable independiente y el coeficiente tipificado mide el impacto relativo de dos o más variables independientes, sobre la variable dependiente (De la Garza García, J. 2013)

\section{Conclusiones}

De acuerdo a los resultados presentados es posible responder la hipótesis del estudio, realizar un análisis en cuanto al objetivo de la investigación, y hacer contribuciones según la investigación conceptual y los resultados obtenidos en la regresión lineal.

La hipótesis se considera aceptada según los resultados estadísticos obtenidos.

En cuanto al objetivo del presente artículo se concluye que sí se cumplió puesto que se definieron las prácticas y roles del equipo líder; con los resultados obtenidos en la regresión lineal se observa que estas prácticas 
impactan positivamente en los resultados de las empresas de la ZMM que están realizando innovación en sus procesos.

Finalmente es conveniente realizar algunas reflexiones con base en los resultados obtenidos y con ello destacar la contribución al conocimiento de la presente investigación:

Las empresas innovadoras han desarrollado un conjunto de prácticas y competencias en su sistema de gestión; estas prácticas realizadas en la cotidianidad son las que mantienen alineada a la organización en la consecución de su razón de ser y objetivos.

Es el equipo líder o la alta administración de las empresas los responsables de desarrollar un sistema de gestión para la innovación que contenga las prácticas que orienten su operación para el logro de los resultados esperados.

La alta administración deben considerar en sus sistemas de gestión, los ítems considerados en la encuesta de la presente investigación, puesto que en ella se reúnen los roles, prácticas, métodos, mecanismos que a su vez también son coincidentes en diferentes empresas innovadoras.

\section{Referencias}

Afuah, A. (1999). La dinámica de la innovación organizacional: El nuevo concepto para lograr ventajas competitivas y rentabilidad. México: Oxford University Press, Inc.

Chesbrough, H. (2006). Open innovation: the new imperative for creating and profiting from technology. Boston: Harvard Business School Publishing Corporation.

Chesbrough, H. V. (2006). Open innovation: Researching a new paradigm. Oxford: Oxford University Press.

Christensen, C. M. (1999). El dilema de los innovadores. Buenos Aires: Ediciones Granica.

Comité Intersectorial para la Innovación. (2011). Programa nacional de innovación. México, D. F: Secretaría de Economía.

CONACYT (2008). Bases de organización y funcionamiento del Registro Nacional de Instituciones y Empreresas Cientificas y Tecnológicas. México, D.F: Diario Oficial.

De la Garza García, J., Morales Serrano, B.N., González Cavazos, B.A. (2013). Análisis estadístico multivariante. México, D.F: Mc Graw Hill.

Garza Villegas, J. (2009). Factores reconstructivistas críticos determinantes de la innovación y efectividad organizacional: Estudio de Empresas de Nuevo León (Tesis doctoral). Monterrey, N.L. México: Tesis FACPYA, UANL. 
Goleman, D. K. (2009). El espiritu creativo. Barcelona: Zeta Bolsillo.

Gundling, E. (2000). The 3M way to innovation. Japón: Kodansha International Ltd.

Instituto Mexicano de Ejecutivos de Finanzas, A.C. (2010). Análisis estratégico para el desarrollo de la MIPYME en México. México, D.F: IMEF.

Kuczmarski, T. (1997). Innovación, estrategias de liderazgo para mercados de alta competencia. Bogotá: Mc Graw Hill.

Kuczmarski, T. (2013). Kuczmarski Innovation. Obtenido de Innovation Culture el 14 de julio de 2013 en: http://www.kuczmarski.com/services/innovation-culture/

OCDE. (2006). Manual de Oslo: Guía para la recogida e interpretación de datos sobre innovación. EUROSTAT: Tragsa.

OCDE. (2009). Innovación en las Empresas. París: Biblioteca en línea de la OCDE www.oecd-ilibrary.org.

RAE. (2013). Diccionario de la lengua española. Madrid: Real Academia Española.

Trias de Bes, F. K. (2011). Innovar para ganar: el modelo A-F. Barcelona: Ediciones Urano.

Valdés, L. (2004). Innovación: El arte de inventar el futuro. México, D. F: Grupo Editorial Norma.

Valdés, L. (2008). El dado de 7 caras. México, D. F: Fundación para el Liderazgo e Innovación Estratégica A.C.

Van de Ven, A. H. (2000). El viaje de la innovación: El desarrollo de una cultura organizacional para innovar. México, D. F: Oxford University Press, Inc.

World Economic Forum. (2012). The global competitiveness report 2011-2012. Suiza: SROKundig. 